Nordic Journal of Modern Language Methodology

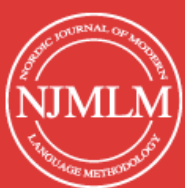

2019, 7 (2), 54-73 Peer reviewed

Temanummer: Spansk i Norge og det norske i den spansktalende verden.

\title{
CONECTANDO MENTES, COMUNICANDO EN ELE
}

\author{
Concha Julián de Vega ${ }^{1}$ \\ Consejería de Educación en el Reino Unido e Irlanda
}

\section{Resumen}

La importancia de la comunicación entre pueblos y culturas es esencial en el mundo globalizado en el que nos movemos. El desarrollo de la predisposición a la comunicación en los procesos de aprendizaje de lenguas es básico para que el aprendiente se anime a utilizar, a jugar y a reflexionar sobre la lengua que está aprendiendo. Establecer las bases para facilitar ambientes de trabajo adecuados que animen al alumnado a usar la lengua meta es uno de los grandes retos a los que el profesorado de ELE se enfrenta, sin lugar a dudas. Esto es así porque la misma naturaleza de las lenguas toca factores afectivos claves que inciden directamente sobre la predisposición de los hablantes a establecer algún tipo de comunicación. Este artículo muestra la importancia de conocer aspectos neurológicos y emocionales, tales como la Disposición a Comunicarse del alumnado de ELE, los procesos cognitivos que forman parte de los procesos de aprendizaje y la influencia de la figura del profesor en el aula, que parecen primordiales cuando se enseña una lengua y fundamentales para que nuestros alumnos lleguen a ser aprendientes independientes en ELE.

\footnotetext{
${ }^{1}$ Concha Julián de Vega es doctora por la Universidad de Sevilla. Actualmente trabaja como asesora técnica en la Consejería de Educación de la Embajada Española en el Reino Unido e Irlanda. Ha sido profesora de lenguas extranjeras en centros de secundaria, en la Universidad de Sevilla y en la Universidad Pablo de Olavide (Sevilla), donde ahora enseña en el Máster de Educación Bilingüe. Fue asesora de formación del profesorado y responsable provincial del Plan de Fomento del Plurilingüismo en la Junta de Andalucía. Pertenece al grupo de investigación ReALL (Research in Affective Language Learning) de la Universidad de Huelva. Investiga sobre los factores afectivos en la enseñanza y el aprendizaje de idiomas, la coordinación docente y el liderazgo en escuelas AICLE (Aprendizaje Integrado de Contenidos y Lenguas Extranjeras) y la aplicación de recursos digitales para la evaluación de ELE. Tiene experiencia como formadora de profesorado, así como en la creación y tutorización de cursos en línea. Ha publicado artículos y elaborado materiales ELE para el Instituto Cervantes y para el Ministerio de Educación, Cultura y Deporte. Es miembro del consejo asesor de las revistas electrónicas TECLA, relacionada con ELE en el mundo anglosajón, y RedELE (Ministerio de Educación, Cultura y Deporte de España).
}

conchjulian@gmail.com 


\section{Nordic Journal of Modern Language Methodology}

Temanummer: Spansk i Norge og det norske i den spansktalende verden.

Palabras clave: ELE; Comunicación; Afectividad; Desarrollo profesional el profesorado; Facilitador; Adquisición de la lengua; Procesos cognitivos

\section{Introducción}

Cuando se habla de enseñanza de lenguas en contextos escolares es inevitable hablar de la figura del profesor y su influencia en los procesos de enseñanzaaprendizaje para alcanzar el objetivo final, que no es otro que el alumnado comprenda, hable y escriba la lengua que se le está enseñando, en este caso, el español como lengua extranjera (ELE). Obviamente esta es una tarea en la que los agentes principales son el profesor y el alumno. En este sentido, del mismo modo que aprendemos a nadar nadando, aprendemos una lengua hablándola, leyéndola y escribiéndola. Esto es, nos tenemos que sumergir en ella de igual manera que nos sumergimos en el agua para aprender a nadar. En la mayoría de las ocasiones, el contacto con la lengua extranjera se posibilita únicamente en el aula. Este es el contexto que permite "nadar" dentro de la lengua meta. El profesor presenta actividades diversas para que el alumno se enganche, comprenda y quiera expresarse en esa lengua. A veces la participación surge de manera espontánea, pero otra es difícil de establecer, sobre todo en los momentos de interacción. Las razones por las que al alumnado le cuesta esa participación pueden ser diversas y la casuística amplia y difícil de abarcar. Existe un punto de inflexión en el que el alumnado decide dar el paso hacia adelante y empezar a jugar, a arriesgarse con la lengua o a permanecer sin intervenir en su zona de confort. Este artículo trata de reflexionar sobre algunos de los elementos afectivos que animan al alumnado a dar ese paso hacia delante, a sumergirse en la lengua y a establecer el puente de comunicación entre profesorado y alumnado en el aula de ELE. Es decir, se tratará el establecimiento de algunas de las condiciones necesarias para que la comunicación tenga lugar dentro del aula y cómo el profesorado puede condicionarlas para que resulte algo natural en nuestros contextos de clase. 
Nordic Journal of Modern Language Methodology

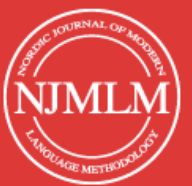

2019, 7 (2), 54-73 Peer reviewed Temanummer: Spansk i Norge og det norske i den spansktalende verden.

\section{La importancia de los contextos de aula}

Earl Stevick, uno de los principales representantes del movimiento humanista en el mundo del aprendizaje de lenguas en los años 70 y 80 , decía que el éxito en el aprendizaje depende menos en los materiales, técnicas y análisis lingüísticos y más en lo que ocurre dentro y entre las personas en el aula (Stevick 44). De igual manera, el modelo socio-cultural de Gardner (2010), nos habla de la influencia en el aprendizaje de lenguas de las microculturas que se originan los contextos del aula. $A$ este respecto, Maclntyre y Mercer (158-159) resumen: El entorno social en el que el aprendizaje tiene lugar es la clave de actitudes positivas y negativas, ya que los alumnos internalizan los elementos del contexto en el que viven. Desde el punto de vista de la psicología y sociología, diferentes estudios han demostrado que el aprendizaje humano está condicionado por las condiciones físicas (Hannaford 96; Jensen 161-174; Mora 33-42). Maslow con su Pirámide o en su teoría psicológica de la jerarquía de las necesidades humanas defiende que conforme se satisfacen las necesidades más básicas, que estarían situadas en la parte inferior de la pirámide, los seres humanos desarrollan necesidades y deseos más elevados, que se situarían en la parte superior de la pirámide. La escala de las necesidades la describe como una pirámide que consta de cinco niveles: En el primer nivel estarían las necesidades fisiológicas, en el segundo nivel se situarían las necesidades relacionadas con la seguridad física. En el tercer nivel estarían las necesidades sociales tales como la amistad, el afecto, la aceptación o la intimidad. El cuarto vendría marcado por los niveles de la estima, confianza, respeto, éxito o reconocimiento. El último nivel sería el de la autorrealización y tendría que ver con el desarrollo de la moralidad, la creatividad, la espontaneidad, la falta de prejuicios, la aceptación de hechos y la resolución de problemas. La idea principal es que mientras nuestras necesidades básicas no estén cubiertas no podremos ocuparnos de nuestras necesidades más altas. En el aula, tenemos que tener en cuenta que si las necesidades fisiológicas, la seguridad, el afecto, y el reconocimiento no están satisfechos, el aprendizaje, si se da, será más lento. En la enseñanza de ELE, por tanto, tenemos que promover espacios en los que el alumno se sienta seguro, esté 


\section{Nordic Journal of Modern Language Methodology}

Temanummer: Spansk i Norge og det norske i den spansktalende verden.

cómodo, para animarlo a experimentar con la lengua meta. Por tanto, los espacios físicos donde el aprendizaje tiene lugar y donde se tienen en cuenta las necesidades fisiológicas del alumnado se hacen fundamentales en contextos de enseñanzaaprendizaje. Por tanto, las herramientas didácticas que ayuden a "controlar" estos aspectos se hacen fundamentales para el profesorado.

Con respecto a los niveles que tienen que ver con las necesidades de seguridad, de aceptación y de autorrealización, el marco del modelo de autoestima que desarrolló Reasoner puede ser muy útil en el aula de ELE. La autoestima podría definirse básicamente como la confianza que tenemos en nuestro propio potencial (Andrés y Arnold 9). Reasoner propone cinco niveles para desarrollar la autoestima: el nivel de seguridad, el nivel de identidad, el nivel de pertenencia, el de propósito y el de competencia (Figura 1).

Niveles de desarrollo de la autoestima

$\mid \begin{gathered}\text { Competencia } \\ \text { Propósito } \\ \text { Pertenencia } \\ \text { Identidad } \\ \text { Seguridad }\end{gathered}$

Figura 1. Niveles de desarrollo de la autoestima (Adaptado de Reasoner 1982)

El desarrollo de la autoestima en procesos de enseñanza de lenguas (Rubio 41-54) implica el garantizar la seguridad física y emocional del alumno en el aula, que los actos y el discurso del profesor deben ser coherentes, que el respeto y la retroalimentación positiva es fundamental, así como el tratamiento del error como parte integrante del proceso de aprendizaje de una lengua. El sentido de identidad tiene que ver con el desarrollo de la autoimagen que el alumno tiene de sí mismo. Para ello, el profesor de lenguas puede proponer actividades donde se puedan desarrollar esa conciencia de uno mismo desde modelos positivos y de aceptación. 
Temanummer: Spansk i Norge og det norske i den spansktalende verden.

El sentido de pertenencia al grupo es fundamental, especialmente con grupos de adolescentes. Actividades grupales de cooperación y colaboración donde los alumnos se integren y sus contribuciones sirvan para una meta común se hacen fundamentales en las dinámicas de nuestras clases. Los últimos niveles de la pirámide estarían relacionados con la cuarta y quinta dimensión que propone Reasoner: propósito y competencia. El sentido de propósito está relacionado con los objetivos que nuestro alumnado se ha propuesto con relación al aprendizaje de la lengua meta. Propone Rubio (41-54) que el profesor como facilitador puede ayudar a crear objetivos realistas o a subdividir tareas para alcanzar metas finales. Por último, el desarrollo del sentido de competencia se alcanzaría cuando nuestro alumnado sintiera haber alcanzado los objetivos que se propuso y desarrollara otros nuevos para avanzar en fluidez con respecto a la lengua extranjera, en este caso, al español. El uso de portafolios, actividades de autoevaluación y evaluación por pares y en grupo proporcionarían información sobre sus logros y el avance hacia nuevos objetivos. El reconocimiento y los incentivos por parte del profesor se hacen imprescindibles en este último estadio en el aula de ELE.

Por tanto, los factores afectivos que se generan antes, durante y después de los procesos de aprendizaje en las aulas tienen tanta importancia como los aspectos cognitivos que se establecen a la hora de adquirir lenguas. Con respecto a esto, Dörnyei, Henry y Muir (66) hablan sobre la incidencia del clima del aula en contextos de aprendizaje de lenguas y la motivación del alumnado. Dörnyei aporta un marco de actuación y diferencia tres niveles. El nivel de la lengua, relacionado con valores culturales, intelectuales y pragmáticos de la lengua en sí; el nivel del aprendiente, donde su autoestima y las características propias de su personalidad y experiencias juegan un papel fundamental en la motivación subjetiva; y a nivel de la situación de aprendizaje que comprendería elementos motivacionales relacionados con los materiales, las programaciones, las tareas, etc., otros relacionados con el profesor: su personalidad, su forma de ser en el aula, su estilo de enseñanza y, por último, con elementos relacionados con el grupo, con la microcultura de aula que se genera. Cuando este mismo autor habla del enfoque orientado hacia procesos en el aula de lenguas extranjeras como modelo generador de motivación, comenta que en 


\section{Nordic Journal of Modern Language Methodology}

Temanummer: Spansk i Norge og det norske i den spansktalende verden.

la primera fase de este enfoque se deben dar las condiciones básicas motivacionales para que haya efectividad, es decir, hace referencia a crear el ambiente adecuado para que el aprendizaje tenga lugar. Los factores que influirán serían el comportamiento del profesor, un clima de apoyo y una cohesión de grupo con normas pertinentes a la situación.

Por tanto, el clima que se genera en el contexto de aula y que influye en el aprendizaje parece que está directamente relacionado con factores afectivos que tenemos que tener en cuenta. Jensen (71) dice que el lado afectivo del aprendizaje es un juego entre lo que sentimos, hacemos y pensamos. No hay separación entre la mente y las emociones. Las emociones, el pensamiento y el aprendizaje están unidos. De la misma manera, el neurobiólogo Schumann (239) cuando habla sobre los elementos afectivos en el aprendizaje de lenguas, hace hincapié en que la mente, la emoción y los procesos cognitivos se diferencian, pero son inseparables. Desde una perspectiva neuronal, el elemento afectivo es una parte integral del proceso cognitivo. Por tanto, aquellos espacios afectivamente positivos ayudarán al cerebro a adoptar condiciones que favorecerán el aprendizaje.

\section{El alumno de ELE y su Disposición a Comunicarse (DAC)}

Aparte del contexto donde tiene lugar los aprendizajes, la voluntad del alumno por aprender una lengua es básica a la hora de establecer puentes de comunicación. Cada alumno trae unas circunstancias (sociales, de familia, culturales, etc) al aula y tenemos que considerarlas para que se animen a intervenir, a sumergirse en el español, ya que solo participarán si ellos así lo deciden. Maclntyre, Dörnyei, Clément y Noels (545-546) sostienen que cuando se define la Disposición a Comunicarse (DAC) debe de entenderse tanto la predisposición del alumno a utilizar la lengua meta como medio de comunicación, como el simple hecho de intentar hacerlo. Consideran este constructo en su conjunto, es decir, estarían incluidas aquellas ocasiones en las que, por ejemplo, un alumno levanta la mano para pedir el turno de palabra, aunque no use al final la lengua meta porque un compañero intervino antes que él. La DAC tiene lugar o no independientemente de la competencia lingüística 


\section{Nordic Journal of Modern Language Methodology}

Temanummer: Spansk i Norge og det norske i den spansktalende verden.

del alumnado. Hay alumnado con alto grado de competencia lingüística que no quiere comunicarse y existe alumnado con competencia mínima que sí quiere hacerlo. Normalmente, el profesor "computa" la situación en el aula en décimas de segundo y automáticamente establece las bases. ¿Qué ocurre cuando la situación es más compleja de lo habitual? El profesor necesitará echar mano de sus conocimientos sobre los factores afectivos, sociales y cognitivos que tienen lugar en los procesos de aprendizaje. Maclntyre et al. (558) plantean que la DAC no es solo un rasgo de personalidad, como se creía en los años 80 (McCroskey and Baer 3-11) sino que cambia según la situación del hablante. Su modelo conceptual se basa en dos tipos de contextos: uno variable y otro que se mantiene más estable, con una serie de elementos generadores que inciden en la DAC y que nos pueden dar claves para generar instrumentos para nuestras aulas (Figura 2).

\begin{tabular}{|c|c|}
\hline & Elementos generadores de DAC \\
\hline \multirow{4}{*}{$\begin{array}{l}\text { Contexto } \\
\text { variable }\end{array}$} & Uso de L2 \\
\hline & Disposición a Comunicarse \\
\hline & Objetivo de comunicación \\
\hline & $\begin{array}{c}\text { Autoconciencia de competencia comunicativa } \\
\text { Ansiedad situacional } \\
\text { Participantes, situación, propósito, tema, canal de comunicación } \\
\text { (oral/escrito) }\end{array}$ \\
\hline \multirow[t]{3}{*}{$\begin{array}{l}\text { Contexto } \\
\text { estable }\end{array}$} & $\begin{array}{c}\text { Motivación } \\
\text { Papel social en un grupo } \\
\text { Sentido de pertenencia a un grupo } \\
\text { Sentido de seguridad } \\
\text { Clima } \\
\text { Actitudes } \\
\text { Creencias }\end{array}$ \\
\hline & $\begin{array}{c}\text { Competencia comunicativa real } \\
\text { (lingüística, discursiva, pragmática, sociocultural, estratégica) }\end{array}$ \\
\hline & $\begin{array}{c}\text { Personalidad } \\
\text { Contexto social }\end{array}$ \\
\hline
\end{tabular}

Figura 2. Elementos generadores de DAC. (Adaptado de Maclntyre et al. 547) 


\section{Nordic Journal of Modern Language Methodology}

Temanummer: Spansk i Norge og det norske i den spansktalende verden.

La Disposición a Comunicarse nos dice que no solo la adquisición de la lengua y la competencia lingüística de un hablante son importantes, sino que también están implicados factores individuales y socio-contextuales, y todos ellos interactúan en el momento que un hablante quiere intervenir en un acto de habla. Teniendo en cuenta lo dicho, desde el punto de vista de nuestras aulas podría ser necesaria una "preparación física y psicológica" a la comunicación por parte del profesor. Por tanto, en el aula ELE, como en cualquier aula de lenguas extranjeras, la preparación para que la comunicación tiene que tener en cuenta tanto los elementos físicos como los cognitivos y afectivos.

\section{Cerebro y cognición en el aula de ELE}

Francisco Mora (25), neurobiólogo de la Universidad Complutense de Madrid y de la Universidad de lowa en EEUU, incide en la importancia de que el profesor sepa de los procesos que tienen lugar en nuestro cerebro cuando aprendemos. Utiliza el término "neuroeducación" y la define, advirtiendo que aún no se le puede considerar una disciplina académica, como una nueva visión de la enseñanza basada en el cerebro [...] Es tomar ventaja de los conocimientos sobre cómo funciona el cerebro integrados con la psicología, la sociología y la medicina en un intento de mejorar y potenciar tanto los procesos de aprendizaje y memoria de los estudiantes como enseñar mejor a los profesores (Mora 25). La neuroeducación trataría de abrir puertas al conocimiento de los procesos cerebrales que tienen que ver con la emoción, con la curiosidad y con la creatividad. Si se aplica esto a los procesos de enseñanza, serviría para la mejora de la práctica docente y los procesos de aprendizaje. Como dice Francisco Mora (28) los profesores debemos ser conscientes de que lo que se enseña es algo más profundo que los propios conocimientos que se transmiten. Por tanto, conocer la estructura física de nuestro cerebro, sus partes, y su funcionamiento durante los procesos cognitivos es fundamental para comprender los procesos de enseñanza-aprendizaje. La visión más coherente para analizar estos procesos debe ser holística porque holística es la manera de trabajar de nuestro cerebro. La neurobiología además nos ha 
Temanummer: Spansk i Norge og det norske i den spansktalende verden.

demostrado, en contra de lo que se creía hace unas décadas, que nuestro cerebro no es estático, sino que va cambiando con el paso de los años según los procesos de aprendizaje a los que lo sometamos. Es más plástico en niños, pero puede modificarse a cualquier edad.

Las investigaciones sobre cómo procesa la información nuestro cerebro no son nuevas. Desde los años 50 han ido surgiendo diversos paradigmas que han ayudado a comprender los procesos cognitivos que tienen lugar en nuestro cerebro cuando aprendemos. Uno de los más influyentes en educación han sido las taxonomías o clasificaciones de este tipo de (Bloom; Anderson y Krathwohl). En los ochenta, la teoría de las Inteligencias Múltiples de Gardner revolucionó los enfoques inmovilistas del aprendizaje. Este investigador define de nuevo el concepto de inteligencia como: el conjunto de destrezas que capacitan a una persona para que pueda solucionar problemas reales a los cuales se enfrenta durante la vida. En un primer momento definió siete (la inteligencia lingüística, la matemática, la visualespacial, la interpersonal, la intrapersonal, la naturalista y la cinestésica) y luego añadió una más: la existencialista, que se refiere a aquellos individuos que aprenden y se manejan en el contexto del "gran panorama" de la existencia humana y se hacen preguntas sobre las razones de nuestro papel en el mundo. Lo novedoso de este paradigma es que defiende que los individuos tienen diferentes grados de competencia en cada una de las inteligencias y que todas se pueden desarrollar y aprender. Otra teoría relacionada con los procesos cognitivos es la teoría Triárquica de la Inteligencia (Sternberg) propone tres tipos de capacidades o inteligencias. La inteligencia analítica, que permite evaluar, comparar y asociar hechos 0 conocimientos de manera lineal. La sintética que tiene que ver con creatividad e intuición y nos capacitaría para imaginar, descubrir y proyectar ideas o planes. Por último, la práctica que sería esencial para llevar a cabo las ideas y proyectos.

Dentro del campo de la lingüística aplicada, también se han investigado los diferentes estilos de aprendizaje cognitivos, conductuales o sensoriales (Reid 87111, Oxford 10-87) que ayudan al profesor de lenguas a comprender los diferentes procesos que tienen lugar en nuestras aulas y a adaptar su actuación y su facilitación al alumnado según las circunstancias que se le presentan. Reid (87) 
Temanummer: Spansk i Norge og det norske i den spansktalende verden.

define a los estilos de aprendizaje como la manera natural, habitual y preferida que el individuo muestra para absorber, procesar y retener información y adquirir destrezas. Las clasificaciones que se han realizado de los estilos de aprendizaje por diferentes investigadores (Reid 87-111) se podrían agrupar en estilos de aprendizaje cognitivos, conductuales (Kolb y Kolb, 6) y sensoriales. Si bien es importante para el profesor tener esta información, es aún más el interpretar estos estilos como flexibles y variables a lo largo del tiempo y considerar que no etiquetan, sino que ayudan al individuo a entender su tendencia, sus preferencias dentro de la gama de estilos. Otro aspecto importante es que los estilos se pueden aprender a desarrollar a través de la intervención educativa, esto es, no son estáticos. Francisco Mora (4041) aconseja no encasillar a los individuos en clasificaciones ya que el aprendizaje, como el mecanismo del mismo cerebro, es holístico.

Cuando elaboremos actividades para nuestra aula de ELE sería aconsejable tener en cuenta todo este conocimiento para tratar la diversidad en nuestras aulas. Por regla general, los sistemas educativos permiten al profesorado abordar la adaptación del aprendizaje desde una doble perspectiva. En primer lugar, el marco general de la macroadaptación, nos aporta la división de la enseñanza en cursos escolares y en asignaturas, con adaptaciones curriculares significativas o no significativas. Esto no deja de ser un puro formalismo que ayuda a estructurar la organización escolar dentro de los sistemas educativos pero que, en la mayoría de las ocasiones, especialmente en edades tempranas, nada tiene que ver con el desarrollo de los procesos de aprendizaje de los niños. En segundo lugar, el profesor aborda el aprendizaje desde una microadaptación, que es lo que ocurre en el aula cuando se atiende a la diversidad individual que presenta cada grupo de alumnado. Si consideramos los estilos de aprendizaje sensoriales en el aula de ELE, nos encontramos con alumnos con tendencia a ser visuales, auditivos y/o cinestésicos. El aprendiente visual aprende más rápido con lo que ve y lo recuerda de manera más natural. Le gusta ver los textos escritos, las fotos, los diagramas, etc. El auditivo prefiere obtener la información escuchando, por ejemplo, a través de conversaciones, pódcasts, grabaciones, etc. Al cinestésico le gusta obtener información por medio del tacto, el gusto, el olfato, con el movimiento de su cuerpo y 


\section{Nordic Journal of Modern Language Methodology}

Temanummer: Spansk i Norge og det norske i den spansktalende verden.

con lo que experimentó (Revell y Norman 31). Por regla general, somos mezclas de todos ellos. En el aula de ELE, a través del juego de la lengua, el profesor puede tener los estilos de aprendizaje presentes y facilitar una variedad de actividades para que el alumnado encuentre su zona de confort al aprender la lengua, en otras palabras, que esté dentro de sus estilos de aprendizaje favoritos. Al presentar diferentes opciones de actividades, los estudiantes no solo refuerzan sus estilos, sino que tienen la oportunidad de desarrollar aquellos en los que son más deficitarios. Un valor añadido de trabajar estos aspectos en el aula de ELE es que el alumnado tiene la oportunidad de desarrollar su competencia de aprender a aprender, al hacerse más consciente de sus procesos de aprendizaje.

Sin embargo, no solo deberíamos tener en cuenta los estilos desde el punto de vista del alumnado, sino desde el punto de vista del profesorado, ya que se tiende a elaborar y presentar actividades dentro de la preferencia o tendencias favoritas, la mayoría de las veces de manera inconsciente, porque están dentro de nuestra zona de confort. Salir de ella, ayudaría a proveer de una diversidad enriquecedora al aula de ELE.

\section{El papel de la emoción}

Cuando tratamos el aprendizaje de lenguas, si bien la parte cognitiva es esencial, lo es también nuestra parte emocional. Oxford (12-16) apunta que nuestro cerebro es emocional y crea relaciones entre el pensamiento, la emoción y la motivación. Desde el punto de vista de la neurociencia, se defiende que las emociones son la base más importante sobre la que se sustentan todos los procesos cognitivos de aprendizaje y memoria. De hecho, Francisco Mora (41-42) añade poéticamente que la emoción es la energía que mueve el mundo, como su propio nombre lo indica: e-moción. Según este investigador, cuando aprendemos las emociones encienden la curiosidad y mantienen la atención y con ello el interés por el descubrimiento de todo lo que es nuevo. Curiosidad y atención son los dos ingredientes básicos para el aprendizaje. El cerebro límbico es la parte principal de nuestro cerebro que se ocupa de estos procesos. Los estímulos sensoriales (ver, oir, tocar, oler, paladear) son analizados 


\section{Nordic Journal of Modern Language Methodology}

Temanummer: Spansk i Norge og det norske i den spansktalende verden.

por la corteza cerebral de forma neutra, esto es, sin significado emocional alguno, para luego pasar por el filtro del sistema emocional. Así lo explica Mora (42):

[...] es allí donde a esas percepciones sensoriales, ya creadas, se las acuña con la etiqueta de bueno o malo, atractivo o rechazable, interesante o soso. $Y$ es después cuando esa información, ya coloreada con ese significado emocional, pasa a las áreas de asociación de la corteza cerebral donde se construyen los procesos mentales, de razón y pensamiento, y se elaboran las funciones ejecutivas complejas. Y también pasa por el hipocampo donde se registra la traza mnemónica (de memoria) de lo percibido y aprendido. En otras palabras, los abstractos o ideas, con las que trabajan las cortezas de asociación para crear el pensamiento ya están impregnadas de emoción. Cognición-emoción es, pues, un binomio indisoluble que nos lleva a concebir de cierto que no hay razón sin emoción.

Gráficamente se podría representar con la figura 3.

Procesos cognitivos y emocionales

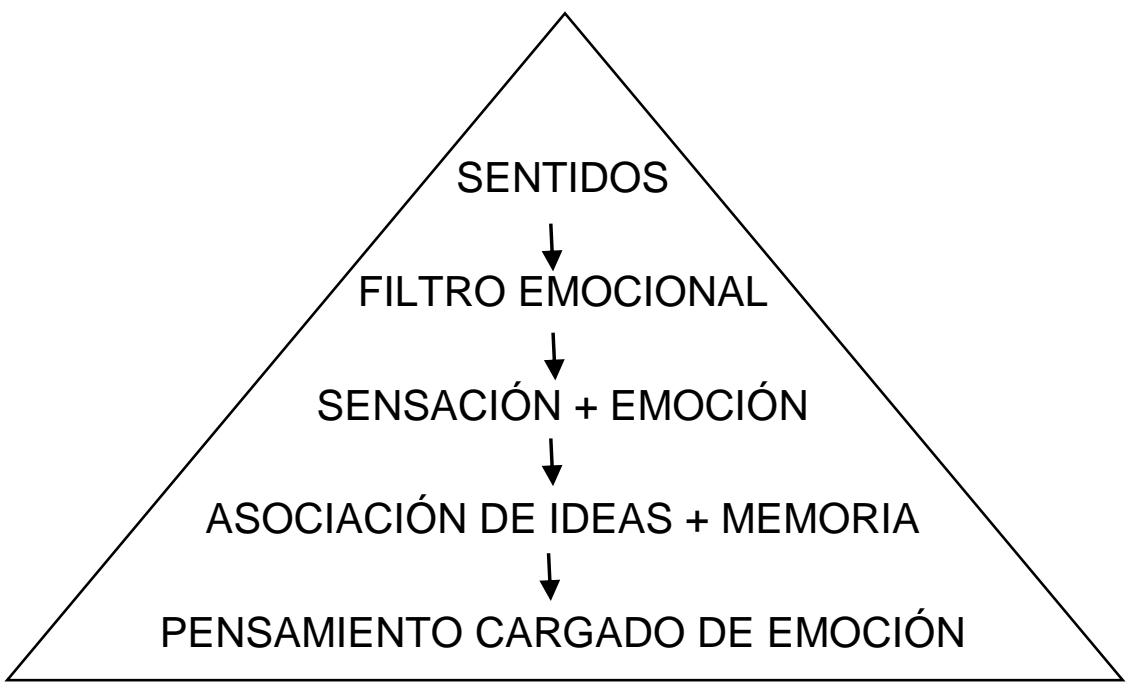

Figura 3. Procesos cognitivos y emocionales (Adaptado de Mora 42)

\section{El profesor en el aula de ELE}

En gran medida, es el profesor el que influye en la creación de estos espacios y en la Disposición a Comunicarse cuando fomenta la curiosidad y la creatividad, cuando 


\section{Nordic Journal of Modern Language Methodology}

Temanummer: Spansk i Norge og det norske i den spansktalende verden.

tiene en cuenta cómo funciona nuestro cerebro, los aspectos cognitivos y los emocionales como partes integrantes de los procesos de aprendizaje que van a tener lugar en el aula. Visualmente se podría representar con la siguiente figura 4:

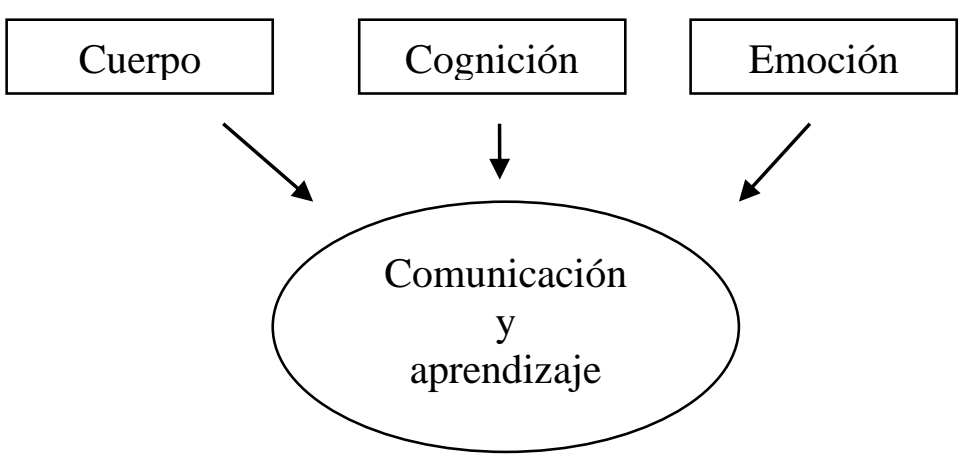

Figura 4: Aspectos para fomentar la comunicación en el aula de ELE.

A modo de analogía con las recetas de cocina, en nuestro caso, el profesor de ELE es el chef que busca los ingredientes, los prepara encima de una mesa, los mezcla, los cocina y los presenta en el plato culinario final. Para que la comunicación tenga lugar y el alumnado se anime a intervenir en ELE, hay previamente que focalizar el momento en el que se buscan los ingredientes que hacen falta para el buen desarrollo de nuestras clases. El momento en el que elaboramos la mezcla apropiada al contexto del grupo y a la situación de aprendizaje que se nos presenta para que nuestros alumnos tomen las riendas de su aprendizaje y se zambullan en la lengua extranjera.

\section{La figura del facilitador}

De todo lo anteriormente dicho, se desprende que el profesor aparece como un "hacedor", un "facilitador" de situaciones, de momentos de aprendizaje. Según Adrian Underhill (130) el profesor facilitador no solo debe tener los conocimientos de la asignatura y su metodología, sino que además debe desarrollar la capacidad de generar el clima de aula proclive a procesos de aprendizaje de calidad. Esta nueva ecuación incluye, por una parte, las relaciones que se establecen entre alumnos y entre alumnos y profesor, el grado de seguridad que sientan los individuos para 


\section{Nordic Journal of Modern Language Methodology}

animarse a participar en el aula, y, por otra, la sensibilidad del profesor para las dinámicas que se establecen, la calidad de la escucha, la aceptación, la posibilidad de participar sin que haya juicios de valor, o el cubrir las necesidades de autoestima de los aprendientes. También tiene que ver con la toma de decisiones conjunta dentro del contexto del aula, el cómo, el qué y el quién, y el reflexionar sobre el por qué. Por tanto, la presencia del profesor tendrá más o menos entidad dependiendo no solo de su faceta técnica sino también de cómo proyecte su dimensión afectiva en el aula, de la forma de hablar, de escuchar, de la forma en que ejerce su autoridad, de su atención a los procesos que ocurren en el grupo, de las actitudes y de las creencias.

\section{Las creencias del profesor}

En las aulas, deberíamos dejar a un lado nuestras creencias, profesionales y personales, no deberíamos asumir nada, porque los mapas de vida que cada participante trae al aula son diferentes $y$, a veces, tendemos a actuar y a juzgar a nuestro alumnado basándonos en suposiciones que arrancan de nuestras creencias. Como profesores está en nuestra mano el ser conscientes de estos procesos y ver más allá. Nuestra labor es compleja porque el desarrollo del currículo no es lineal, ni secuencial, sino cíclico, y está marcado por las creencias que el profesor trae al aula acerca de la educación y del aprendizaje (Burns 55-56). A este respecto Cummins (33-51) apunta que en el campo del aprendizaje de lenguas se debería analizar con más detalle cómo los profesores conciben los procesos de aprendizaje, qué términos, imágenes y constructos suelen utilizar, cómo los interpretan y los resuelven en el día a día. Es decir, cómo se establecen los nexos entre las creencias metodológicas, los comportamientos en el aula, el pensamiento profesional y la práctica de la enseñanza.

\section{La empatía en el aula de ELE}

Profesor y alumnos son dos partes inseparables de una misma pieza y la conexión que se establezca entre ellos configurará los procesos que tengan lugar a lo largo 


\section{Nordic Journal of Modern Language Methodology}

Temanummer: Spansk i Norge og det norske i den spansktalende verden.

del tiempo. Establecer rapport, una sintonía favorable al aprendizaje es necesaria para la clase de ELE. En el aprendizaje, los sentimientos que experimentamos encienden la curiosidad por descubrir lo que es nuevo y mantienen la atención, y con ello, son fundamentales para almacenar esa información en nuestra memoria (Mora 73-79). La empatía es un ingrediente esencial que entra en juego para alcanzar la sintonía en el aula, tanto por parte del profesor hacia los alumnos, como entre los mismos alumnos. Batson, Ahmad y Lishner (418) dicen que a la empatía no se le puede considerar una emoción sino un conjunto de sentimientos donde entrarían la compasión, la ternura y la identificación por lo que le ocurre al otro. Esto puede ser positivo o negativo, pero para que haya empatía siempre se le deseará lo mejor. La empatía es situacional, depende del contexto e influye en el aprendizaje porque interviene en los procesos de maduración del cerebro humano (Mora 50). Mercer (99-101) apunta que, aunque la empatía es importante en la educación en general, existen algunos aspectos que son especialmente relevantes en el campo del aprendizaje de lenguas extranjeras. En ese sentido argumenta que para hablar una lengua extranjera hay verdaderamente que dotarse de una nueva identidad. Es necesario ponerse en la piel de los hablantes de la otra lengua. Además, existen investigaciones que demuestran que aquel alumnado con mayores niveles de empatía presenta mejor aptitud para la pronunciación de la lengua meta (Guiora, Paluszny, Beit-Hallahmi, Catford, Cooley y Dull 48; Dewaele y Wei 352-366). El uso de una lengua requiere de interacción con los demás. En esta interacción es donde compartimos nuestros pensamientos, sentimientos, preocupaciones, esperanzas y deseos, y estrechamos lazos. Durante el proceso de hacerse entender y de entender a los demás, bien escuchando, hablando o escribiendo, nuestro alumnado tiene la oportunidad de desarrollar la competencia comunicativa en ELE. Actividades donde se practique la escucha activa, la reflexión, el ponerse en lugar del otro, las canciones, las actividades donde aparezca el humor, los proyectos, los juegos y concursos en parejas y en grupo pueden ser algunas de las tareas de aula que fomentarían la empatía entre alumnos y entre alumnos y profesor. 
Nordic Journal of Modern Language Methodology

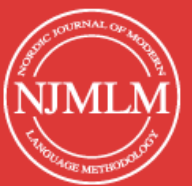

2019, 7 (2), 54-73 Peer reviewed Temanummer: Spansk i Norge og det norske i den spansktalende verden.

\section{El discurso del profesor de ELE}

$\mathrm{Si}$, desde el punto de vista del alumnado, la clave de la adquisición de una lengua estriba en su Disposición a Comunicarse y su uso efectivo de dicha lengua, desde el punto de vista del profesor la llave para conformar contextos favorecedores del aprendizaje son las palabras, el tono o los gestos que use en su discurso. El discurso en el aula, en su sentido amplio, es uno de los principales recursos que tenemos para establecer la conexión, la sintonía necesaria para abrir la puerta a la comunicación y a los procesos de aprendizaje, ya que es el discurso uno de los canales por los que se transmite la emoción. Con él captamos la atención de nuestro alumnado y creamos el ambiente que invite a la interacción. Mora (67) apunta que, si el discurso del profesor va cargado de emoción, por cómo se utiliza o cómo se entona, si los conceptos, a veces complejos, van conexionados de significado emocional, lo que se aprende se hará de manera más sólida y duradera. ArnoldMorgan y Fonseca-Mora (110-112) hablan de la importancia del discurso afectivo en el aula de lenguas para generar empatía, y proponen ejemplos prácticos tales como el aprender los nombres del alumnado desde el primer contacto con el grupo, como una forma de construir el sentido de identidad en el aula. Gorham (50-51), para favorecer el buen clima, recomienda el uso de "nosotros" y "nuestro", en lugar de "vosotros" y "vuestro", así como preguntar por la opinión al alumnado para llegar a acuerdos en las dinámicas de clase. Underhill (132-135) apunta a la parte no verbal de la comunicación en el aula de lenguas como uno de los aspectos que se relegan. El tono de nuestras palabras, el contacto visual, la expresión en nuestro rostro o la proximidad pueden marcar diferencias a la hora de crear ambientes de trabajo positivos. Por tanto, las palabras en sí y el modo en que se expresen son el vehículo del conocimiento y de la emoción, y esto es especialmente importante para los que enseñamos lenguas porque somos los expertos que presentamos el juego de las palabras, de las estructuras, de los textos escritos o hablados en el aula. Con ellas y con nuestros actos conformamos los espacios donde tendrá lugar el aprendizaje. El crear las condiciones precisas y necesarias en cada momento está en nuestras manos. 


\section{Nordic Journal of Modern Language Methodology}

Temanummer: Spansk i Norge og det norske i den spansktalende verden.

\section{Conclusión}

La importancia de establecer una buena comunicación en el aula, en su sentido más amplio, es uno de los pilares básicos cuando abordamos los procesos de enseñanza-aprendizaje de una lengua, en nuestro caso, del español como lengua extranjera. Para ello, hay que tener en cuenta la influencia de factores relacionados con las condiciones físicas, nuestro cuerpo y cerebro, con los procesos cognitivos y con los emocionales. Estas son las bases desde las que se pueden cimentar contextos favorables al aprendizaje de la lengua meta. Aquí, tanto el alumnado, como el profesorado son las dos caras de una misma moneda. Si bien, la influencia de la figura del profesor en el aula hace que este sea la clave para construir estos ambientes positivos que propicien el aprendizaje de ELE. Su aproximación e interpretación a las necesidades físicas, cognitivas y emocionales del alumnado promoverá o no a que los procesos de aprendizaje tengan lugar de manera efectiva. Debemos tomar la temperatura emocional a nuestras aulas, gestionando diálogos para promover la empatía, compartiendo objetivos y estableciendo acuerdos, usando recursos para crear seguridad, para que el alumnado se sienta grupo, para que alcance sus metas y las celebre. Así mismo, debemos considerar las necesidades de nuestro cerebro cuando procesamos una lengua y cuando la estamos aprendiendo. Debemos pensar cómo podemos influir para que, al hacer saltar la chispa de la curiosidad, se mantenga suficientemente la atención para que la nueva información pase a los mecanismos de la memoria. No solo los conocimientos de la lengua y su metodología son importantes, debemos tener en cuenta que, como dice Francisco Mora (51), la emoción, los sentimientos, sus mecanismos cerebrales y su expresión en la conducta siguen siendo la base, el pilar esencial, que debemos conocer para construir un edificio sólido en la enseñanza, en nuestro caso, en la enseñanza del español como lengua extranjera. 
Nordic Journal of Modern Language Methodology

2019, 7 (2), 54-73 Peer reviewed Temanummer: Spansk i Norge og det norske i den spansktalende verden.

\section{Bibliografía}

Andrés, Verónica y Arnold, Jane. Seeds of Confidence. Self-esteem Activities for the EFL Classroom. Rum: Helbling Languages, 2009. Impreso.

Arnold, Jane y Fonseca-Mora, M.Carmen. "Affect in Teacher Talk". En Tomlison, Brian (ed.) Language Acquisition. London: Continuum, 2007. Impreso.

Batson, Daniel, Ahmad, Nadia y Lishner, David. "Empathy an Altruism”. En López, Shane. y Snyder, C. R. The Oxford Handbook for Positive Psychology. New York: Oxford University Press, 2011. Impreso.

Bloom, Benjamin (ed.) Taxonomy of Educational Objectives, Handbook I: Cognitive Domain. New York: Longman, 1956. Impreso.

Anderson, Lorin y Krathwohl, David (eds.). A taxonomy for learning, teaching, and assessing: A revision of Bloom's taxonomy of educational objectives. New York: Longman, 2001. Web. 12 Sep 2017.

Burns, Anne, "Teacher Beliefs and their Influence on Classroom Practice". Prospect 7 (1992). Impreso.

Cummins, A. "Student Teachers' Conceptions of Curriculum: Towards an Understanding of Language Teacher Development". En TESL Canada Journal 7 (1989). Web. 30 Agosto 2017.

Dörnyei, Zoltán. Motivational Strategies in the Language Classroom. Cambridge: Cambridge University Press, 2006. Web. 3 Sep 2017.

Dörnyei, Zoltán, Henry, Alaistair y Muir, Christine. Motivational Currents in Language Learning. Frameworks for Focused Interventions. Routledge (2016). Impreso.

Dewaele, Jean-Marc y Wei, Li.“Multilingualism, Empathy and Multicompetence”. En International Journal of Multilingualism 9(2012) 4. Web. 10 enero 2018

Gardner, Howard. Inteligencias Múltiples. La teoría en la práctica. Madrid: Editorial Paidós, 1995.Impreso.

Gardner, Robert. Motivation and Second Language Acquisition: The SocioEducational Model. New York: Peter Lang, 2010. Impreso.

Guiora, Alexander, Paluszny, Maria. Beit-Hallahmi, Benjamin, Catford, John, Cooley Ralph y Dull, Cecelia Yoder. "Language and Person Studies in Language Behaviour". En Language Learning 25 (1975) 1. Web. 15 dic. 2017 
Nordic Journal of Modern Language Methodology

2019, 7 (2), 54-73 Peer reviewed

Temanummer: Spansk i Norge og det norske i den spansktalende verden.

Hannaford, Carla. Smart Moves. Atlanta: Great Ocean Publishers, 1995. Impreso.

Jensen, Eric. Teaching with the Brain in Mind. Alexandria, VA: Association for Supervision and Curriculum Development, 1998. Web. 11 oct. 2017.

Jensen, Eric. Brain-based Learning. San Diego, CA: The Brain Store, 2000. Impreso.

Kolb, David. The learning style inventory. Boston: McBer, 1976. Impreso.

Kolb, David. Experiential learning: Experience as the Source of Learning and Development. Englewood Cliffs, NJ: Prentice-Hall, 1984. Impreso.

Kolb, Alice Y. y Kolb, David A. The Kolb Learning Style Inventory-Version 3.12005 Technical Specifications. Philadelphia: HayGroup, 2005. Impreso

Maclntyre, Peter y Mercer,Sarah. "Introducing Positive Psychology to SLA". En Studies in Second Language Learning and Teaching 4 (2014) 2, 153-172. Web. 15 dic. 2017.

Maclntyre, Peter, Dörnyei, Zoltan, Clément, Richard y Noels, Kimberly. "Conceptualizing Willingness to Communicate in a L2: a Situational Model of L2 Confidence and Affiliation". En The Modern Language Journal 82 (1998) 4, 546-562. Web. 12 Sep 2017.

Maslow, Abraham. Motivation and Personality. Nueva York: Addison-Wesley Longman, 1987. Impreso.

McCroskey, James and Baer, Elaine. "Willingness to Communicate: the Construct and its Measurement". Ponencia presentada en Annual Concention of the Speech Communication Association, Denver, C.O. (1985) Web. 10 enero 2018.

Mercer, Sarah. "Seeing the Wold through Your Eyes: Empathy in Language Learning and Teaching". En Maclntyre, Peter, Gregersen, Tammy y Mercer, Sarah. Positive Psychology in SLA, 2016. Impreso.

Mora, Francisco. Neuroeducación. Madrid: Alianza Editorial, 2017. Impreso.

Oxford, Rebecca. "Toward Psychology of Well-Being for Language Learners: The "EMPHATICS" Vision". En Maclntyre, Peter, Gregersen, Tammy y Mercer, Sarah. Positive Psychology in SLA, 2016. Impreso.

Reasoner, Robert. Building Self-esteem: a Comprehensive Program. Palo Alto, CA: Consulting Psycologist Press, 1982. Impreso.

Reid, Joyd. Learning Styles in the ESL/EFL Classroom. Boston: Heinle \& Heinle, 1995. Impreso. 


\section{Nordic Journal of Modern Language Methodology}

2019, 7 (2), 54-73 Peer reviewed Temanummer: Spansk i Norge og det norske i den spansktalende verden.

Revell, Jane. \& Norman,Susan. In Your Hands. London: Saffire Press, 1998. Impreso.

Rubio, Fernando. "Self-esteem and Self-concept in Foreign Language learning". En Mercer, Sarah y Williams, Marion. Multiple Perspectives on the Self in SLA. Bristol: Multilingual Matters, 2014. Impreso.

Schumann, J.. "Where is cognition?" Studies in Second Language Acquisition 16 (1994). Web. 16 Oct. 2017

Sternberg, Robert Jeffrey. "A Triarchic View of Giftedness: Theory and Practice". En Coleangelo, Nicolas y Davis, Gary (eds.). Handbook of Gifted Education. Boston, MA: Allyn and Bacon, 1997. Impreso.

Stevick, Earl. Teaching Languages: $A$ Way and Ways. Rowley, MA: Newbury, 1980.Impreso.

Underhill, Adrian, "Facilitation in Language Teaching". En Arnold, Jane. Affect in Language Learning. Cambridge, UK: Cambridge University Press,1999. Impreso. 